\title{
Differential Models for Integrated Drought Risk Management
}

\author{
Xinghua Fan (Corresponding author), Dan Zhao \& Lixin Tian \\ 301 Xuefu Road, Faculty of Science, Jiangsu University \\ Zhenjiang 212013, Jiangsu, China \\ E-mail: fan131@ujs.edu.cn
}

\begin{abstract}
We exam the Vitae System from a mathematic point of view. Taking communities' capacity, disaster factor and management efforts or policies into account, a differential model for Integrated Drought Risk Management is introduced in this paper. The effects of the management strength on the water consumption are studied first on a constant then on a periodic background. They are slow-fast model and chaotic one, respectively. Geometric and numerical method are applied to those models.
\end{abstract}

Keywords: Differential system, Integrated risk management, Drought management, Vitae system, Slow-fast model, Chaos

\section{Introduction}

The Integrated Disaster Risk Management (IDRM) plays a key role in Natural Disaster Reduction. The Vitae System Model was presented by Okada as a conceptual framework for IDRM (Okada, N., 2006; Okada, N., 2004; Jiquan, Zhang, 2007, p19-23). It claims to view cities, regions and communities as vital integrity with robustness and resiliency in its coping capacity. Based on the Vitae System Model, Okada suggests that disaster planning and management problems be integrated with urban planning and management ones in a unified framework (Fig. 1).

There are some research works on the Vitae System framework (Okada, N., 2008; Xu, Wei, 2008, p59-65). In (Xu, Wei, 2008, p59-65), some basic model for disaster shelter planning and evaluation based on the Vitae system were established. In (Okada, N., 2008), within the framework of Vitae System for IDRM, the questions of resources allocation under high uncertain in a community factor and among communities were considered, and the disaster recovery process was interpreted.

The main purpose of this paper is to set an analysis model for IDRM. This kind of attempt has not been read before. We choose drought disaster mitigation management for the convenience of quantifying. On one hand, the relevant factor involved in disaster mitigation management can be chosen as water consumption while water consumption in a city and region has been documented in quantitative fashion. On the other hand attempts have been made to control or mitigate drought by many governments.

The rest of this paper is organized as follows. In Section 2, we present the Integrated drought risk management model which is a general three-dimension differential system. Two special cases of the model are considered in the next two sections. In Section 3, under a constant risk, geometric method is applied to analyze the water consumption trend of different management efforts. Numerical method is used in Section 4 under a period risk. The last section is the conclusion.

Insert $<$ Figure $1>$ here

\section{Integrated drought risk management model}

We will display in detail how the general differential model for Integrated drought risk management is established in this section.

Consider a city or region faced with rapid population growth and uncertain climate future challenges such as drought. As a Vitae System, three basic ingredients can be considered as the community's coping capacity capability, disaster risk factor and the management factor. While in (Okada, N., 2006), the community's coping capacity capability is 10 day average water saving percentage, we select water consumption. The two variables are inverse proportioned.

Let $w(t)$ be the total water consumption of the whole city at time $t$. If there are no disaster nor management effort carried in the region, the rate of change $\frac{d w}{d t}$ is simply the natural consumption rate $G$ which is here assumed to be a function of water consumption only. It is nature when there is no water or consumption reaches the maximal water reserve $W$, the 
consumption rate must be zero. Let us consider what the function looks like. The Logistic model is the simplest one (Fig. 2).

In the Logistic model, $\frac{d G(w)}{d w}<0$, that means the consumption rate per unit water consumption $\frac{G(w)}{w}$ is a decreasing function of water consumption $w$. However, sometimes it is possible that the consumption rate per unit water consumption $\frac{G(w)}{w}$ is first increasing with $w$ then decreasing. The shape of $G(w)$ is first convex then concave. As a special case, in the limit, $G(w)$ is first initially negative then positive. Then we get the different types of natural consumption rate functions as shown in Fig. 2. This kind of function can be seen in other fields (Gatto, M., 1987).

Insert $<$ Figure $2>$ here

Disaster risk function can be measured in different ways. We suggest it is the damage or possible damage the disaster causes to cities, measured in money. $r$ can be quite complex.

Let $m$ represents, in suitable unites, the amount of labor and capital invested.In real world situations $M$ may be a rather complex function of $w, r, m$. When management policy and disaster factor are considered, the rate of change $\frac{d w}{d t}$ of water consumption is the difference between natural consumption rate and them.

Based on the above analysis process, we present the following differential model for IDRM

$$
\left\{\begin{array}{l}
\frac{d w}{d t}=G(w)-a r-b m, \\
\frac{d r}{d t}=R(w, r, m), \\
\frac{d m}{d t}=M(w, r, m),
\end{array}\right.
$$

where $a$ and $b$ are constants representing the impact strength the disaster risk and the management have on the coping capability.

System (1) is quite subtle. In order to know its actually evolution, every function and parameter must be given. This is a big challenge. However in many occasions what we want to know is just the trend not the exact mathematical numbers. Thus in the coming sections we will only study two special cases.

\section{Slow-fast Vital model}

Assume the city or region is always under the threat of drought, that is $r(t)=$ const.. So the management function $M$ is specified as a function of water consumption and the disaster risk strength, i.e. $M=M(w, m)$. Since the draught exists as a constant, it is reasonable to take a steady policy to deal with the drought. Mathematical speaking, the rates at which the management function evolute in Vitae System can be much slower than that of the water consumption. Hence we rewrite $M=\varepsilon \tilde{M}(w, m)$. The value of $\varepsilon$ can be determined in such a way that the maximum absolute values of $\frac{d w}{d t}$ and $\tilde{M}(w, m)$ are the same.

$$
\left\{\begin{array}{l}
\frac{d w}{d t}=G(w)-a r-b m, \\
\frac{d m}{d t}=\varepsilon \tilde{M}(w, r, m),
\end{array}\right.
$$

System like (2) is usually called "slow-fast" system (Rinaldi, S., 2000, p507-521). Given the initial conditions, when $\varepsilon$ is very small trying to integrate the system (1) is not easy, even with the most powerful simulation software. That is because it is almost impossible to keep numerical errors under control when dealing simultaneously with numbers differ by a few orders of magnitude. Fortunately Geometric Analysis provides an effective way to treat this kind of problem (Rinaldi, S., 2000, p507-521). The singular perturbation approach allows one to study the dynamics of the system in a very clear and appealing geometrical form. (1) is first studied in the singular case $\varepsilon=0$ which is usually a bifurcation analysis. When $\varepsilon$ is very small, the real solution differs less than $\varepsilon$ from the singular solution.

In the limit case $\varepsilon=0$, we get $\frac{d w}{d t}=G(w)-a r-b m$, then $m=\frac{G(w)-a r}{b}$. The relation between $m$ and $w$ is just a linear transformation. So we can get the state variable bifurcation graph from the consumption rate functions.

Trajectories can be obtained from bifurcation graph even if one does not know the exact function $\tilde{M}$ but knows only where $\tilde{M}$ is positive and where it is negative. If $\tilde{M}$ is positive, $m$ increases and the singular trajectory develops from the left to the right, whereas it develops in the opposite direction if $\tilde{M}$ is negative.

\subsection{Logistic case}

Let $w_{1}$ and $w_{2}\left(w_{1}<w_{2}\right)$ be the roots of $G(w)-a r-b m=0$. They represent the smallest and highest water consumption respectively. Fig. 3 shows the bifurcation diagram when the consumption rate is Logistic. A stable equilibrium (upper solid line) collides with an unstable equilibrium (dashed line) at the critic value $m^{*}$ of the parameter. The arrows in the figure indicate the time evolution of the water consumption. Longer arrow means faster evolution. Assume the smallest consumption $w=w_{1}$ be a stable equilibrium for all values of $m$. Thus, for $m<m^{*}$ there are two attractors, and their basins of attraction are separated by the unstable equilibrium (dashed line); whereas for $m>m^{*}$ there is only one global attractor (the smallest consumption). 
Insert $<$ Figure 3, Figure $4>$ here

A tiny perturbation $(\Delta)$ of the critic value $m^{*}$ induces a large shift of the state of the system. Suppose that $m$ is slightly smaller than $m^{*}$ and that the system is at its stable equilibrium (solid line)and that $m$ is increased by a small amount $\Delta$ such that $m+\Delta>m^{*}$. After such a perturbation the consumption will start moving downward. At first, this motion is very slow. But after some time, the motion becomes fast and the consumption goes down to the smallest state. Such a shift in dynamic systems may have large economic or social consequences.

Suppose the management effort is strengthened very slowly, the consumption will vary accordingly. From the bifurcation diagram, we can see the consumption variable move slowly to the right along the solid line until the fold point is reached, then drop vertically (fast transient) to the lower stable branch, and finally, continue to move to the right along the lower branch. Thus, the time evolution of the water consumption can be obtained as in Fig. 4. The consumption remains higher for a long time, then it drops to the smallest in a relatively short time and finally remains at the smallest level forever. It is of no significance to strengthen the management any more when the management strength has already reached the critical value $m^{*}$.

\subsection{Critical case: Vital rhythms}

Fig. 5 shows the bifurcation diagram when the consumption rate is critical. The solid lines indicate stable equilibria, whereas the dashed line represents an unstable equilibrium. The system is bistable because it has two alternative stable equilibria for $m^{*}<m<m^{* *}$.

Insert $<$ Figure 5-8 $>$ here

Consider the situation in which the managing authority adjusts its regulation adaptively in such a way that management is enhanced if water consumption exceeds a certain limit. As shown in Figs. 6 and 7, this regulation can be interpreted as the isocline of zero change in management $\left(\frac{d m}{d t}=0\right)$ separating the area of increasing from that of decreasing consumption.

In the tight case (Fig. 6), the management isocline intersects the bifurcation diagram at the upper branch, resulting in a unique stable equilibrium $E$ with relatively large consumption from any initial state, the system converges to this water consumption equilibrium through a series of fast and slow transitions, as illustrated by two singular trajectories in the figure. Note that the first trajectory is composed of one fast $Q \rightarrow Q^{*}$ and one slow $Q^{*} E$ phase, whereas the second trajectory is composed of four alternate fast (double arrows) and slow (single arrow) phases. On the other hand, a strict management, such that the dotted isocline intersects at the lower branch of the bifurcation diagram, would result in a similarly unique low consumption stable state.

In the loosen case, the isocline separates the two stable pieces of the bifurcation diagram (Fig. 7), the system converges to a cycle from any initial state (Fig. 8). Such cycles are characterized by periods of relatively little change, separated by rapid dramatic transitions in the consumption. For that reason such cycles are called slow-fast cycles. Such slow-fast cycles were observed in the form 10-day average water saving percentage in (Okada, N., 2006) and were called Vital rhythms.

\section{Chaotic model}

If $M=$ const . and $r=$ const, the water consumption has the fast transition and the relaxation cycles. It is reasonable for both the disaster risk and the management to change. Climatic changes periodically. Assume the risk is periodic and the management changes according to the consumption and management itself. In slow-fast system, Van der Pol system is a famous equation, and for simplicity we assume the management differential function is linear. We obtain the following integrate system under periodic risk.

$$
\left\{\begin{array}{l}
\frac{d w}{d t}=w-w^{3} / 3-a r-b m, \\
\frac{d r}{d t}=f \cos (\omega t), \\
\frac{d m}{d t}=c w+\alpha-\beta m,
\end{array}\right.
$$

Notice that (3) can be rewrite as a planar system which is chaotic for some special parematers (Ramesh, M., 2001, p23952405). The integrate disaster system can be chaotic under some cases. Taken $f=0.74, \alpha=0.07, \beta=0.08, c=0.1$, we get a chaotic attractor as shown in Fig. 9. The consumption varies unpredictable with the management as shown in Fig. 10. There are many researches on chaotic system (Ramesh, M., 2001, p2395-2405).

Insert $<$ Figure 9, Figure $10>$ here

\section{Conclusion}

We gave a differential system as the model for integrated drought risk management. When the risk was taken to be constant, this model turned to be a slow-fast system. Geometric analysis was applied to it. Various kind of water consumption trends under different management were displayed. Under a period risk background, the differential model might be chaotic for some system parameters.

The mathematical models were our beginning study on quantifying the Vitae system. We took a short-cut approach to 
the problem by only researching the constant and periodic risk backgrounds. Further studies such as practical simulation, model explanation and applications are needed.

\section{Acknowledgements}

The authors would like to thank Professor Norio Okada who raised the problem. Research is supported by the Postdoctoral Foundation of China (No. 20080441071), the Post-doctoral Foundation of Jiangsu Province (No.0802073c) and the High- level Talented Person Special Subsidizes of Jiangsu University (No. 08JDG013).

\section{References}

Gatto, M. \& Rinaldi, S. (1987). Some models of catastrophic behavior in exploited forests. Vegetatio, 69, $213-222$.

Okada, N. (2006). City and region viewed as vitae system for integrated disaster risk management. Annuals of Disas. Prev. Res. Inst., Kyoto Univ., No. 49 B, (79B).

Okada, N. \& Fang, L. (2008). A methodological challenge towards sustainable management of environment and disaster risks. Presentation at the 4th Japan-China Joint Seminar on Sustainable Manegemanet of Cities and Regions under Disaster and Environmental Risks.

Okada, N. \& Tatano, H. ( 2004). A japan's challenge towards anticipatory and participatory urban disaster risk management: case study of tonankai earthquake disaster initiative. IUPEA Conference, Louisville, USE.

Ramesh, M. \& Narayanan, S. (2001). Chaos control of bonhoeffer-van der pol oscillator using neural networks. Chaos Solitons Fractals, 12, 2395-2405.

Rinaldi, S. \& Scheffer, M. (2000). Geometric analysis of ecological models with slow and fast processes. Ecosystems, 3, 507-521.

Xu, W., Okada, N., Xu, X. \& Shi, P. (2008). Conceptual model of disaster shelter planning based on the vitae system. Journal of Catastrophology, 23, 59-65.

Zhang, J., Zhang, H. \& Okada, N. (2007). Integrated urban disaster risk management: an innovative approach and challenge in the 21st century. Human Geography, 19-23.

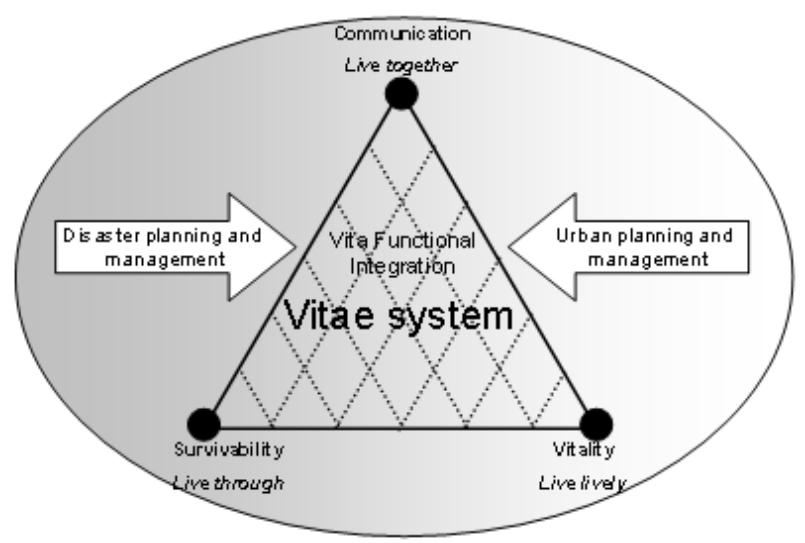

Figure 1. Integrated Urban (Reginal) Management Viewed as Vitae System [2] 


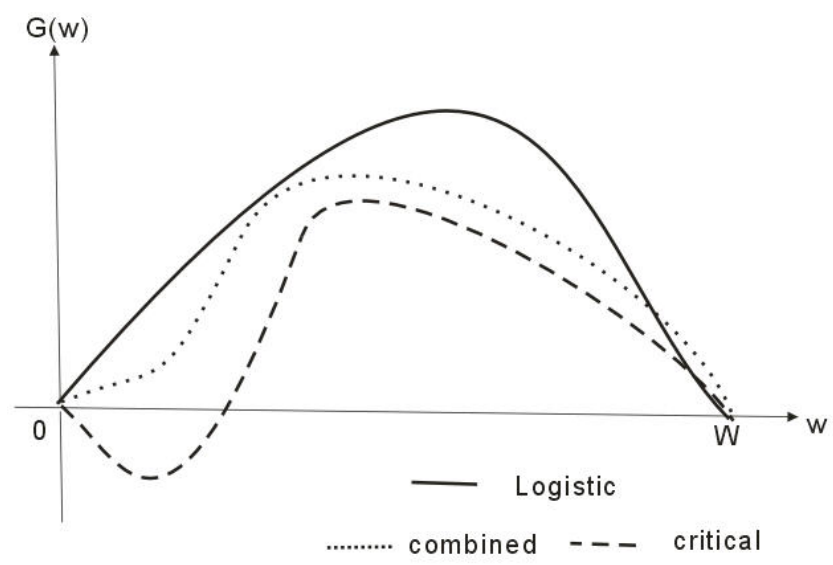

Figure 2. Different types of natural consumption rate functions

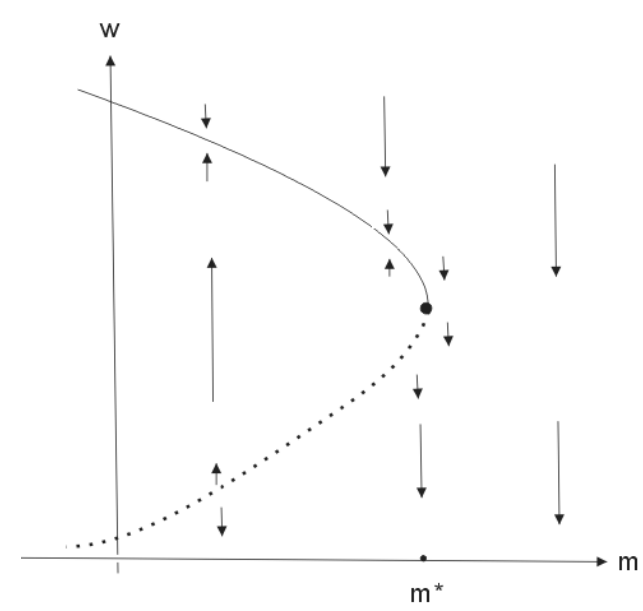

Figure 3. Logistic case

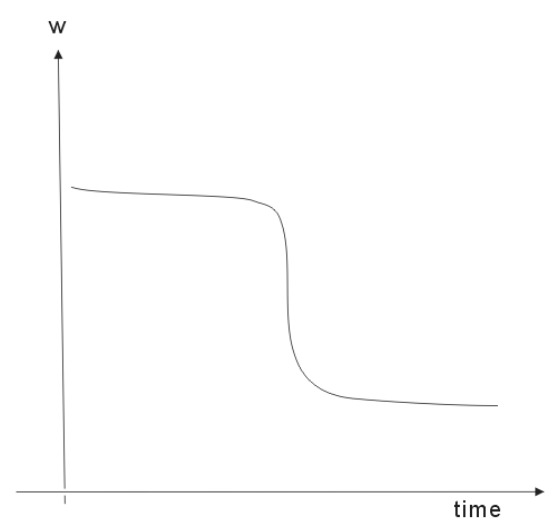

Figure 4. Water consumption to time 


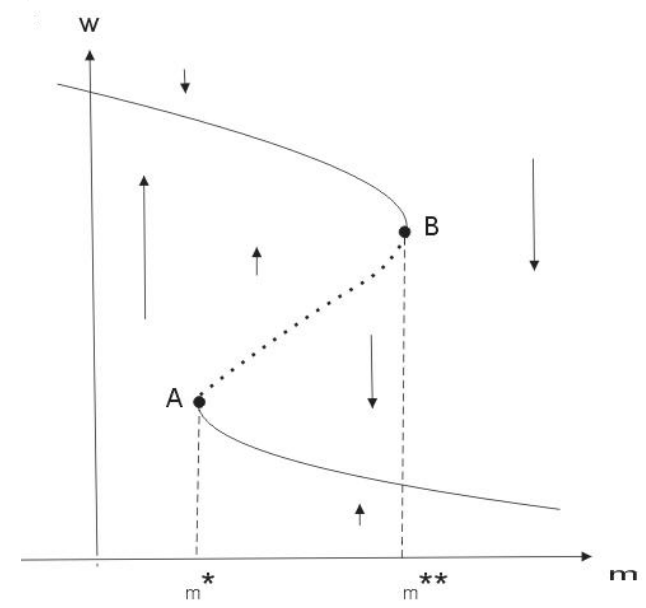

Figure 5. Bifurcation diagram for critical case

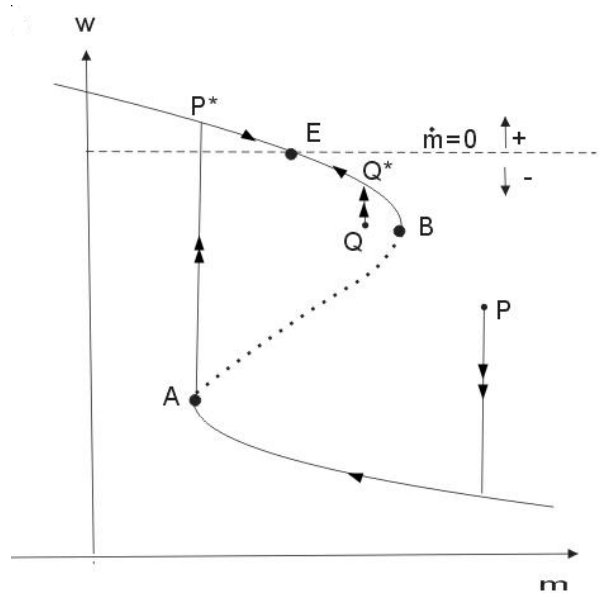

Figure 6. Tight management cause water consumption to a stable equilibrium

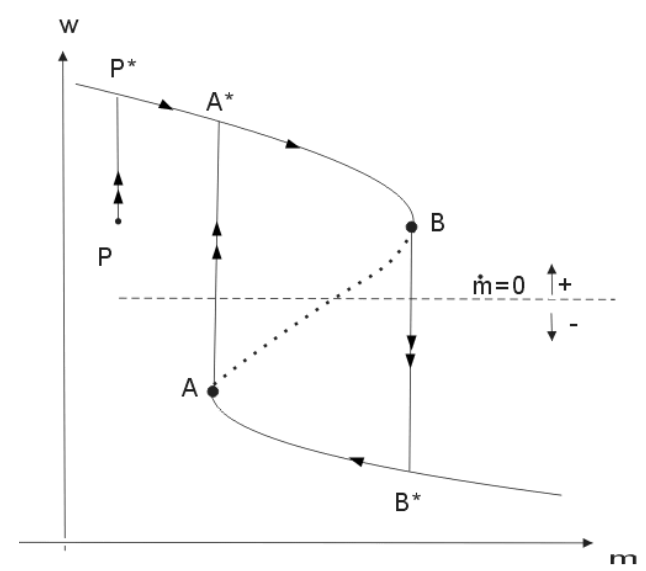

Figure 7. Loose management 


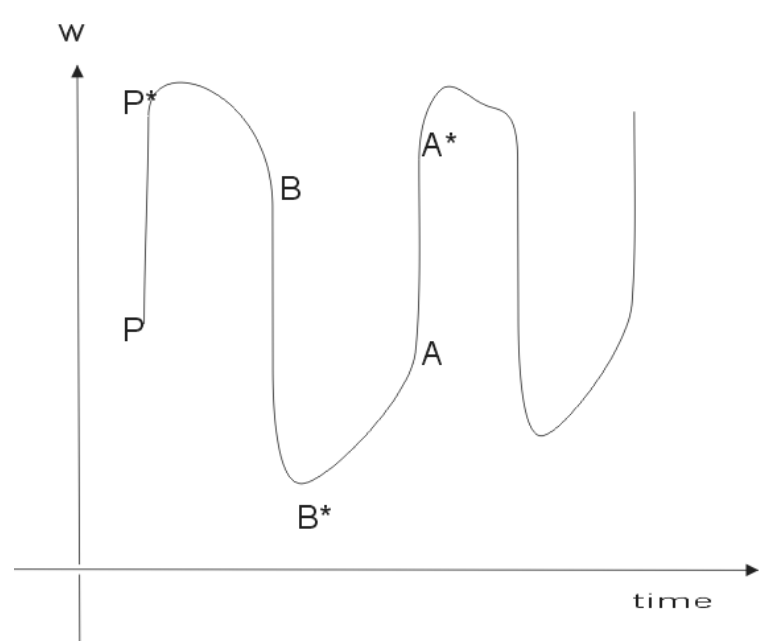

Figure 8. Vital rhythms

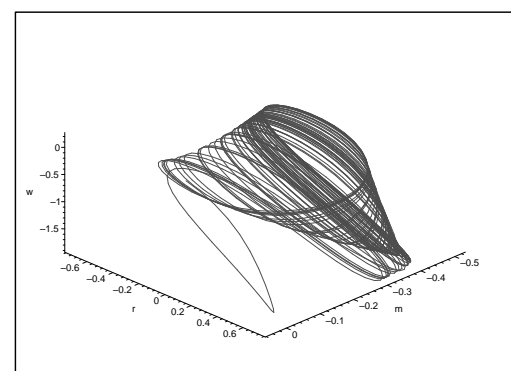

Figure 9. Three-dimension state phase

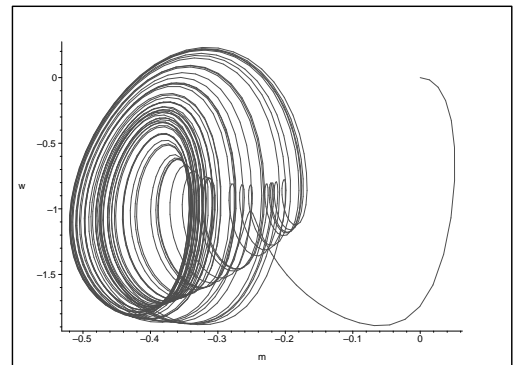

Figure 10. Water-management state phase 